\begin{tabular}{c} 
International Journal of Engineering \& Technology, 7 (3) (2018) 1626-1630 \\
International Journal of Engineering \& Technology \\
SPC \\
Website: $\begin{array}{c}\text { ww. sciencepubco.com/index.php/IJET } \\
\text { doi: } 10.14419 / \text { ijet. } 7 \text { 7i3.14242 } \\
\text { Research paper }\end{array}$ \\
\hline
\end{tabular}

\title{
A-ZHLS: adaptive ZHLS routing protocol for heterogeneous mobile adhoc networks
}

\author{
M. V. Narayana ${ }^{1}$, Aparnarajesh Atmakuri ${ }^{2}$ \\ ${ }^{1}$ Professor, Department of CSE, Guru Nanak Institutions Technical Campus, Hyderabad \\ ${ }^{2}$ Research Scholar, Department of CSE, Vishvesvaraya Technological University \\ *Corresponding author E-mail: mvnarayanacse@gmail.com
}

\begin{abstract}
The routing protocols in MANET are mainly classified into two type's i.e. pro-active and reactive based protocol based on its problems in the network. One of the prime and foremost issues, which have gained a lot of scope, is its dynamic nature of the networks i.e. the stability of nodes is not guaranteed in the network. Hence, this leads to loss of data packets that needs for its retransmission. Many advancements and Novel protocols have been devised in recent years for efficiency and effectiveness in the ad hoc networks. However, hybrid routing proto-cols that inculcates the nature of reactive and proactive has gained a lot of importance. ZHLS is one of the Hybrid routing protocol, and is a two tier hierarchal routing that divides the area into zones in which mobile nodes move randomly. The process of promoting ZHLS protocol is purely based on homogeneous scenarios which mean the nodes in the network are statistically identical, but there is lack of studies for heterogeneous set-up where mobile nodes act in a different way in an identical network. In this paper, we try to propose a novel approach known as "Adaptive-ZHLS: AZHLS" that can be suitable for heterogeneous scenarios. Experimental set up and simulated results will show that AZHLS provides higher packet delivery fraction as compared with the traditional one.
\end{abstract}

Keywords: Bypath routing; Heterogeneous Scenarios; IER; MANETS; ZHLS

\section{Introduction}

Promotion in Technology of Wireless communication has gained a tremendous attention in our day to day activities. To this mobility has added a innovative facet, where the devices such as WLAN cards, Internet sticks, Zig-bee etc. are been carried by the natives to enjoy the services of internet by make the life and work easier. A rising trend in the Wireless network architecture is MANET that can be easily deployed in any environment which is fundamentally different from Static/ wired network as it's requires a base station. Mobile Ad hoc networks have gained a lot of importance in recent years because of its infrastructure less base stations. Every node in the system can host for a communication to take place in the absence of central administrator, between the other nodes or parts of the network. This network needs a relevant hardware support to be an ideal candidate during emergency situations. Because of its limited transmission range, the nodes that are adjacent to each will forward the packets during communication process.

In general, the lifecycle of mobile ad hoc network is neither planned nor is this planning negotiated with any trusted infrastructure. The ad hoc networks elements are diverse in nature, and have a collective aim by enabling the exchange of data and its resources. The mobility of the nodes makes a dynamic topology in a random manner, this temperament of mobile nodes rise to heterogeneous [1] and homogenous scenarios [2].

\subsection{Heterogeneity in mobile devices}

A great deal in the research done so far in MANET is almost based on homogenous scenarios with identical nodes capabilities and its communication characteristics too. However, this type of model lacks in important characteristics of realistic ad hoc networks where nodes having different capabilities in terms of transmission power, data rates, processing capability, robustness and congestion.

The charisma of heterogeneity is that all nodes/devices of the network are not identical in nature, some may act as a server and others can be clients. The great differences in these devices vary from battery capacity, device size, computational power and its memory. These disagreements between the nodes can affect the protocol design and its performance in communication. The methodologies [37] for homogenous cases may not be an efficacy for heterogeneous environment. Moreover, the homogenous ad hoc scenarios may not perform better in terms of scalability of the network. The nodes are realistic in ad hoc networks [8] as in battle field networks, where soldiers carrying devices are portable, and dominant devices carried by tanks, vehicles etc may have different communication capabilities in terms of reliability, power etc. as mentioned above. Such heterogeneity nature of an ad hoc network needs a different power transmission level with varying communication range.

Another realistic nature of ad hoc network can evident in University/ College campus with a wide range of portable and personal system that ranges from laptops, palmtops and most common used ones is Smart phones. Each of this system components are tailored with different capabilities and communication techniques that are intended to use. Mobile apps such as "Share-IT" or the oldest of the technology "Bluetooth" is an energy saving tools with most common used in handheld devices or mobile phones. The merging of these devices into one network can be an innovation of novel applications and services such as "VoIP or Location based services". The integration of these devices starts communicating with each other via a personal device of one PAN to the PAN personal device. An additional trend which emerged in recent years and been promoted 
widely is "Internet of things" where devices are heterogeneous in nature.

\subsection{Motivation}

It is realistically observed that in certain scenarios, the nodes in ad hoc networks may have varying speeds that ranges low to high as discussed in [9]. In ZHLS routing, only one path is constructed to each destination node. When a link in a path is broken, packet delivery is stopped until another path is reconstructed. In heterogeneous cases, the mobility of the nodes are not constant and the communication links are broken relatively more frequently and communication stops until another path is reconstructed. This kind of problem is especially more serious for real time applications or streaming data in which retransmission does not take place. Frequent table update means frequent execution of the Shortest Path Algorithm which is computationally intensive and not desirable. Therefore, there is a need to propose a novel adaptive approach to make ZHLS more effective and efficient and to study the performance of ZHLS in such heterogeneous case.

\subsection{Organization of the Paper}

Section [1] concise the introduction of MANETS, heterogeneous systems and the motivation for the proposed research. Section 2 gives briefly discusses about the various existing methodologies in the heterogeneous systems for Mobile Ad hoc Networks. The conventional ZHLS is briefly described in Section 3. The proposed approach Adaptive-ZHLS is presented in Section 4. Section 5 and Section 6 conclude the paper and references used for this article.

\section{Literature survey}

In this paper [10], X. Du et.al considered two types of nodes to imbibe heterogeneous network one is B-nodes with high data rate and transmission power and the other with G-nodes (Less powerful nodes). The authors proposed a multicasting routing protocol exclusive for heterogeneous scenarios in ad hoc networks with design of novel MAC also known as hybrid MAC(HMAC) which appeared to be efficient than IEEE 802.11b.

S. Yang et.al [11] designed a HLS protocol known as heterogeneous location service to overcome the issue of random nature of nodes. This article also presented a cross-layer approach to make light of the problems caused by asymmetrical links that spans over MAC and network layers of assorted networks.

Yuefeng Huang et.al [12] also developed a cross-layer-based approach to solve the issues of asymmetry links in mesh networks. Hidden and exposed terminal problem in heterogeneous scenarios is been addressed via handshaking and channel reservation mechanism. Apart from this, the article also presents the sharing of topological information with MAC layer by the establishment of reverse paths for unidirectional links.

To identify unidirectional links a novel protocol known as Loosevirtual-Clustering (LVC) has been proposed by Peng Zhao et.al [13] for power aware heterogeneous scenarios in MANETs. A hierarchical structure is maintained and via a clustering, a virtual backbone is created. To improve space utilization and throughput in the network, from each group of nodes a node with high power is chosen as cluster head and avoided the packet forwarding through these cluster heads.

W. Liu et.al [14] designed a framework based on cross layering known as "DELAR: stands for Device-Energy-Load ware relaying" to deal the problems of conserving energy of the devices in the heterogeneous environment of MANETs. This novel approach integrated the energy saving techniques such as power-aware routing, power MAC, Power saving modes and transmission power control. Here also the nodes are classified into high data rates, limited power and unlimited power known as B-nodes, P-nodes etc.

Abolhasan et.al [15] developed a strategy for heterogeneous networks known as "On-demand Utility-Based Routing Protocol
(OUBRP)". This reactive based protocol is implemented over AODV so during routing discovery, the protocol try to choose the nodes with highest resources to reduce the number of message broadcasting by the remaining set of nodes in the network. The protocol tried to improve the discovery of routes in the network there by reducing route failures

In article [16], Xu et al. proposed a MBN routing protocol for nodes with high data rates and transmission power. Ye et al. [17] to assist routing in heterogeneous MANETs they used additional reliable nodes. Papers like [18-19] mainly focused on asymmetrical link for heterogeneous networks.

\section{Conventional ZHLS}

Zone-Based Hierarchical Link State Routing Protocol or ZHLS is a hybrid routing protocol. This Hybrid routing protocol incorporates the features of conventional Reactive and Proactive routing. The methodology of creating zones is based on nodes GPS by using the geographic partitioning. Every node in the network will maintain a database that contains the information about zones. With the help of this database, each node can identify its respective area by mapping its geographical location. In this protocol the network constructs two routing tables one for Intra-zone and other for Inter-zone path construction. The size of the zone can be small or big, it can also be variable or static. Static zone size is defined in static database which stored in nodes memory. However, variable zone size depends on various factors ranging over nodes density to nodes propagation characteristics. In variable size zones, the plot of the network needs to be rebroadcasted whenever s new node joins or leaves the network.

As ZHLS is hybrid protocol and is based on Zone-ID and Node-ID, hence this requires to define two different levels of topology i.e. zone level and node level topology. The zone level arrangement of nodes contains the information about the zones connection and its virtual links within the network. Whereas, the node level arrangement explain about the physical connectivity of the nodes within a zone. Though, this protocol defines two level hierarchies but there is no requirement of cluster heads. Data/ packet routing within the network is established based on Zone-ID and Node-ID of the destination by solving the problem of information delivery during link breaks. The two different topologies of this protocol are represented using Link state packets.

\subsection{Route establishment for intra-zone}

Every node makes a list of its neighbor ID and floods them as Link State Packet within the zone. Neighbors of adjacent zone are also included in this list. The detail procedure is as shown below.

1) If the destination is within the zone then the node broadcast a location request packet.

2) The nodes that receive the location request packet response them via location response packet.

3) Upon receiving the response, the sending node creates a link state packet.

4) The node level topology information is broadcasted to all the remaining nodes within the zone.

After receiving LSP from the rest of the other nodes in the zone, each node makes a Link State Table on the receipt of LSPs to understand the topology of the network. Then the Inter-zone routing table is created with the shortest path algorithm.

\subsection{Route establishment for inter-zone}

A node that is able to communicate with nodes of another zone is called a gateway node. Each node in a zone aware of the gateway node of its residing zone and can communicate with the help of LSP The detail procedure for inter-zone path construction is as shown below.

1) Each node prepares the zone LSP from the node LSPs of the gateway nodes. 
2) Flooding of Link state packets in the network through gateway nodes.

After receiving zone LSPs from other zones, each node saves them in Zone Link State Table and knows the zone level network topology. Here as well, Shortest Path Algorithm is used to create Interzone routing table.

\section{Adaptive ZHLS routing protocol}

It is observed that a uniform zone and a single path construction from source node to destination node in heterogeneous cases might lead to poor performance since some nodes moves very fast (30-40 $\mathrm{m} / \mathrm{s}$ ) with no pause in between while some nodes move relatively slow $(0-10 \mathrm{~m} / \mathrm{s})$ with some pause time in between. This motivated to propose an Adaptive Zone Hierarchal Link State Routing Protocol that functions on differences heterogeneous situations in a single network. The two different heterogeneous circumstances that are considered in this paper are:

1) Different types of mobile node in the same network i.e. high mobile nodes and low mobile nodes.

2) If the path to the destination node is broken down due to the broken link or nodes with high mobility in the path moving away, the packets will not be delivered to the node until a new path is reconstructed

Therefore, to reliably functions for different heterogeneous circumstances, the network topology of Adaptive ZHLS is logically viewed in two diverse topologies provided that these are in the same network zone. The two logically viewed topologies are:

- For the low mobile nodes, a Bypath route is constructed for routing

- For the High mobile nodes, an Inter-Zone Routing Protocol is constructed

\subsection{By-path route construction approach}

In this proposed method, we derive an alternative path for the broken link via peripheral nodes of the path. The node that is used for detour is registered beforehand and when the path is broken, packets are forward via the registered node without waiting for the reconstruction of a new path. Suppose in Figure 1(a), node a and node $\mathrm{b}$ are communicating. If the link $\mathrm{a}-\mathrm{b}$ is broken the communication is detoured via node c. In Figure 1(b), node a and d are communicating via node $b$. When the node $b$ moves away, the communication will continue via node $\mathrm{c}$ without reconstructing a new path. When constructing communication path, neighbor nodes of the nodes on the path are constructed and are registered as detour nodes in routing table. In order to do so an extra field in ZHLS routing table is added as shown in Table 4. In the similar manner, detour zones and nodes are registered for inter-zone routing table as shown in Table 5.

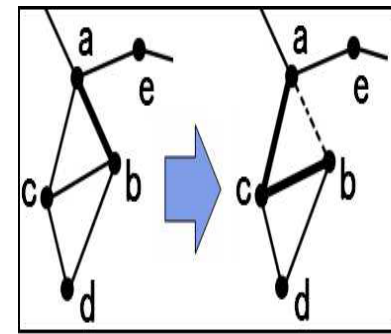

(a)

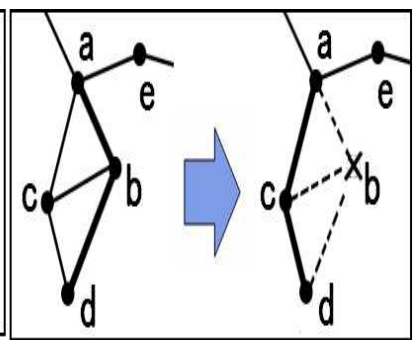

(b)
Fig. 1: An Example of by-Path Routing.

\subsubsection{Intra-zone by-path construction}

In intra-zone by-path probing, a node first selects a neighboring node as a target node to which an alternative path is to be constructed. It then checks its Link State Table and finds a node which is linked to itself and the target node and registers the node as a by- path node between itself and the target node. The procedure for finding a detour path for each target node is given below.

The inquiring node executes the above procedure for all its neighbor nodes and constructs an alternative path for each neighbor nodes. In order to discover an optional path for those neighbor nodes that are two hop away, they are put in a queue. After inquiring all the neighbor nodes of the target node, the nodes in the queue are probed as target nodes. In this case, also, a node, which is linked to both the probing node and the target node, is searched in the Link State Table.

\subsubsection{Inter-zone detour path construction}

An alternative path cannot be constructed for nodes which lie in other zones. This is because ZHLS has hierarchical structures and a node in one zone does not have information of nodes in other zones. Furthermore, there is no mechanism in ZHLS to infer the state of each other's zones. In our proposed method, we use inter-zone routing table and Zone Link State Table to construct a zone level detour path. In order to traverse a zone level path, the packets are in fact forwarded by nodes hop by hop within a zone. Therefore, for zone level path, if the detour path is taken, there is a possibility that the detour path may become much longer than the original path. Thus in our proposed method, we consider the detour path which will have the same number of zone hops as the original path. Moreover, in ZHLS inter-zone routing, packet forwarding occurs by checking the destination node id and its zone id.

Therefore for each node, it is sufficient to know the neighbor node and neighbor zone to grasp the path information to the destination node residing in neighbor zone. Here, in order to construct a detour zone path, we consider only neighboring 8 zones. Construction of a zone level detour path is the same as the construction of detour path for two hops away node in node level detour path. Each node selects one of its neighbor zones and obtains its ZoneLSP. Then it selects one of the two hops away zones registered in ZoneLSP and makes it a target target zone and the zone it is residing in using Zone Link State Table. By checking the routing table, if the searched zone is not already on the route then it is the detour zone to the target zone and is registered as a detour zone.

\subsection{Inter zone routing protocol}

For High Mobile nodes, this Routing Protocol is employed where every node is considered in the zone form with the zone radius zero. When node with high mobile has any data to be sent but it could not find any path to the destination then it broadcast a route request packet (RREQ) using broadcast resolution protocol. The intermediate which receives the request does not have any route then it appends its IP address there by forwarding the packet using BRP. However, if the intermediate node has a route to the destination node then it appends the route to the RREQ packet by creating a response packet to the sender node. The RREP (route reply packet) packet is route along the reversed accumulated route towards the source. If the routing zone is modified then the Inter-zone routing protocol repair and optimize the changed routes.

For each modified route an alternative path is constructed for identifying the shortest path to the destination thereby displacing failed links and suboptimal segments. The format of the packet has an identifier that determines the whether it is route request or route reply packet. Apart from this the packet also contains other fields such as list of IP addresses of the path, source ID, Destination ID, No. of hops. A pointer is used for identification of the node in the list for forwarding purpose.

\section{Experimental result and its analysis}

The Simulation used for evaluation of the protocols is Network Simulator 2 (NS2). The goal of simulation experiment is to examine high and low node mobility in the heterogeneous domain for proposed Adaptive ZHLS routing protocol. The measures employed 
for assessment are (i) Packet Delivery Ratio (PDR), (ii) Total Control Overhead (iii) Average end to end Delay. Table 1 represents the constraints applied for demonstrating the simulation to analyze the Adaptive routing protocol in the heterogeneous domain.

Table 1: Constraints Used for Simulation

\begin{tabular}{ll}
\multicolumn{2}{c}{ Table 1: Constraints Used for Simulation } \\
\hline Parameters & Value \\
\hline Network Size & 1500 X 1500 (m.sq) \\
Transmission Radius & $250 \mathrm{~m}$ \\
Transmission Rate & $2 \mathrm{Mbps}$ \\
& $0-10 \mathrm{~m} / \mathrm{s}$ (Slow Nodes) \\
Node Speed & $30-40 \mathrm{~m} / \mathrm{s}$ (Fast Nodes) \\
Routing Zone Radius & 1 \\
Data Packet Size & $512 \mathrm{MB}$ \\
Simulation Period & $1000 \mathrm{~s}$ \\
\hline
\end{tabular}

The relative investigation of both Conventional ZHLS and Adaptive ZHLS for diverse estimation on Packet Delivery Ratio, Tota Control overhead and Average End-to-End Delay at diverse Mobile heterogeneous Environments of the node as per given in Table 1 is specified. As given in Table 2 and Figure 2, the modification in PDR for Adaptive ZHLS is higher when compared to traditional ZHLS for diverse mobility in the heterogeneous domain.

Table 2: Comparison of Packet Delivery Ratio for Traditional ZHLS and Adaptive ZHLS

\begin{tabular}{|c|c|c|}
\hline \multirow{2}{*}{ Pause Time (sec) } & \multicolumn{2}{|c|}{ Packet Delivery Ratio } \\
\cline { 2 - 3 } & Traditional ZHLS & Adaptive ZHLS \\
\hline 30 & 0.24 & 0.32 \\
\hline 40 & 0.23 & 0.32 \\
\hline 50 & 0.2 & 0.34 \\
\hline 60 & 0.23 & 0.35 \\
\hline 70 & 0.26 & 0.36 \\
\hline 80 & 0.3 & 0.42 \\
\hline 90 & 0.5 & 0.53 \\
\hline 100 & 0.63 & 0.65 \\
\hline
\end{tabular}

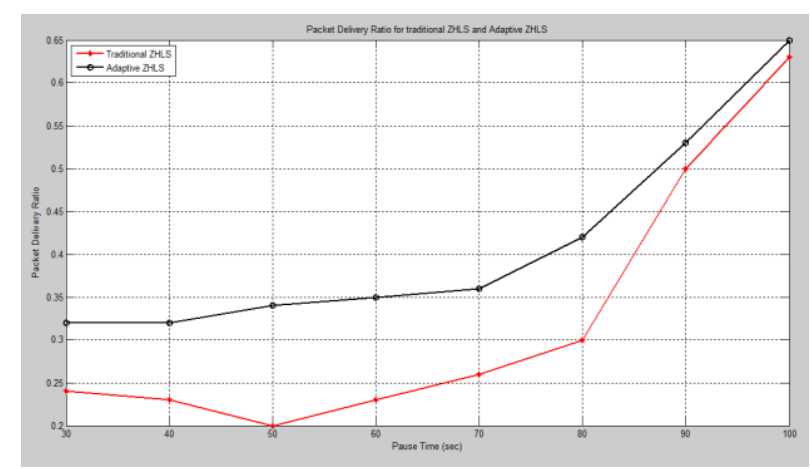

Fig. 2: Comparison of Packet Delivery Ratio for Traditional ZHLS and Adaptive ZHLS.

The effect of average end-to-end delay on the movement of the nodes is given in Figure 3 and Table 3. Outcomes illustrates that the delay aspect is desperately identical even though the pause time is minimum with both higher and lower mobility. At very high mobility circumstance, Adaptive ZHLS provides approximately 5\%-10\% improved outcomes compared to traditional ZHLS might be due to the easy construction of alternative or By-Path route for high mobile nodes.

Table 3: Comparison of End-to-End Delay for traditional ZHLS and Adaptive ZHLS

\begin{tabular}{|c|c|c|}
\hline \multirow{2}{*}{ Pause Time (sec) } & \multicolumn{2}{|c|}{ End-to-End Delay } \\
\cline { 2 - 3 } & Traditional ZHLS & Adaptive ZHLS \\
\hline 30 & 0.5 & 0.49 \\
\hline 40 & 0.42 & 0.42 \\
\hline 50 & 0.37 & 0.34 \\
\hline 60 & 0.3 & 0.26 \\
\hline 70 & 0.15 & 0.12 \\
\hline 80 & 0.04 & 0.02 \\
\hline 90 & 0.08 & 0.06 \\
\hline 100 & 0.09 & 0.05 \\
\hline
\end{tabular}

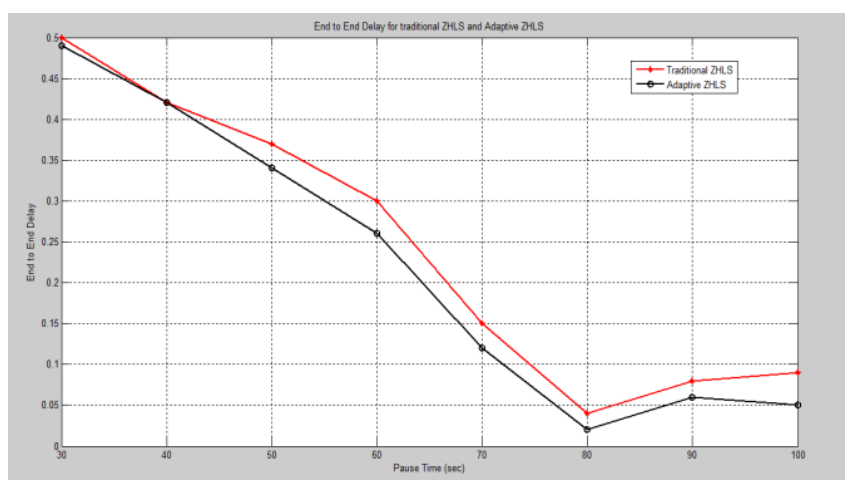

Fig. 3: Comparison of End-to-End Delay for Traditional ZHLS and Adaptive ZHLS

The Figure 4 and Table 4 represents the influence of control overhead on the flexibility of the nodes. There is an enhancement in the minimization of control overhead in the topology owing to Adaptive ZHLS to a maximum of $20 \%$ and a minimum of $10 \%$ in heterogeneous domain. Adaptive ZHLA has employed the benefit of vailability of alternative paths. This minimized the control overhead due to relocation of the novel paths at any time when it is essential. At maximum mobility circumstances, outcomes are evidenced to be worthy with a significant minimization in control overhead to approximately $30 \%$. At lesser mobility circumstances and while the nodes are not mobile, an enhancement of $10 \%$ in the reduction of control overhead is found.

Table 4: Comparison of Total Control Overhead for Traditional ZHLS and

\begin{tabular}{|c|c|c|}
\hline \multirow{2}{*}{ Pause Time (sec) } & \multicolumn{2}{|c|}{ Control Overhead } \\
\cline { 2 - 3 } & Traditional ZHLS & Adaptive ZHLS \\
\hline 0 & 2590 & 2450 \\
\hline 100 & 2310 & 2160 \\
\hline 200 & 2030 & 1790 \\
\hline 300 & 1989 & 1660 \\
\hline 400 & 1720 & 1480 \\
\hline 500 & 1690 & 1340 \\
\hline 600 & 1520 & 1210 \\
\hline 700 & 1450 & 1150 \\
\hline 800 & 1243 & 1020 \\
\hline 900 & 1160 & 1016 \\
\hline 1000 & 1000 & 960 \\
\hline & & \\
\hline
\end{tabular}

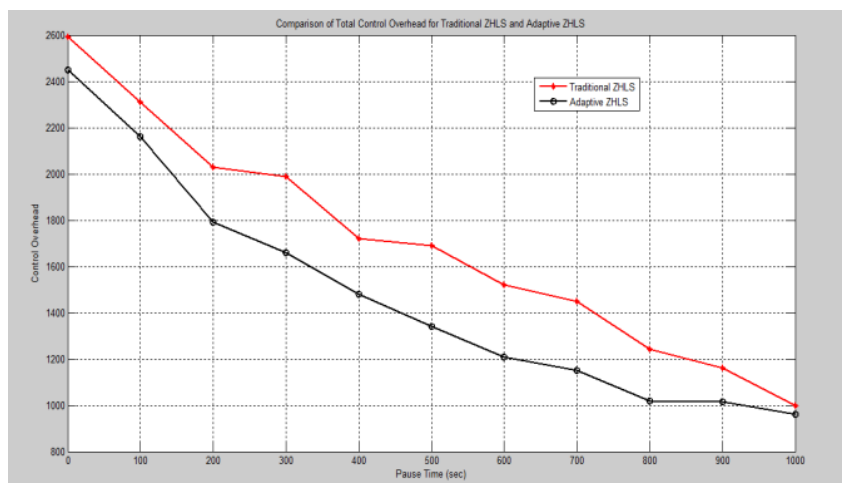

Fig. 4: Comparison of Total Control Overhead for Traditional ZHLS and Adaptive ZHLS

\section{Conclusions}

In this paper, we proposed an Adaptive Zone based Hierarchal Link State Routing Protocol with varying node speeds. From the results, we can conclude that the Conventional ZHLS Routing Protocol in heterogeneous scenarios is not appropriate. Further, the results of both ZHLS and AZHLS has been compared and showed with varying node speeds. The proposed Approach i.e. Adaptive-ZHLS showed the best performance for packet delivery ratio and Control 
routing overhead in all cases where the by-path are constructed for the high mobile nodes and traditional inter zone routing protocol approach is suggested for low mobile nodes in the heterogeneous domain.

\section{References}

[1] J.P. Macker and M.S. Corson, "Mobile ad hoc networking and the IETF", ACM Mobile Computing and Communications Reviews, Vol 4, No. 4, pp. 12-13, October 2000 https://doi.org/10.1145/380516.380529.

[2] C.E. Perkins, "Ad Hoc Networking", Addison-Wesley, Boston, MA, 2001.

[3] E.M. Royer and C.K. Toh, "A review of current routing protocols for ad hoc mobile wireless networks", IEEE Personal Communications, Vol. 6, No. 2, pp. 46-55, April 1999. https://doi.org/10.1109/98.760423.

[4] H. Wu, C. Qiao, S. De and O. Tonguz, "Integrated cellular and ad hoc relaying systems: iCAR", IEEE Journal on Selected Areas in Communications, Vol. 19, No. 10, pp. 2105-2115, October 2001 https://doi.org/10.1109/49.957326.

[5] M. Joa Ng and I. Tai Lu, "A Peer-to-Peer Zone-Based Two Level Link State Routing for Mobile ad Hoc Networks", IEEE on Selected Areas in Communications, Vol. 17, No. 8, 1999, pp. 1415-1425. https://doi.org/10.1109/49.779923 ...

[6] X. Du, D. Wu, W. Liu, and Y. Fang, "Multiclass routing and medium access control for heterogeneous mobile ad hoc networks," IEEE Transactions on Vehicular Technology, vol. 55, no. 1, pp. 270-277, Jan. 2006. https://doi.org/10.1109/TVT.2005.861183.

[7] S. Yang, X. Yang, and H. Yang, "A cross-layer framework for position based routing and medium access control in heterogeneous mobile ad hoc networks," Telecommunications Systems, vol. 42, no. 1/2, pp. 29-46, Oct. 2009. https://doi.org/10.1007/s11235-009-9167-8

[8] Wei Liu; Chi Zhang; Guoliang Yao; Yuguang Fang, "Delar: A device energy-load aware relaying framework for heterogeneous mobile Adhoc networks", IEEE Journal on Selected Areas in Communications, vol. 29, no. 8, pp. 1572-1584, September 2011

[9] Y. Huang, X. Yang, S. Yang, W. Yu, and X. Fu, "A cross-layer approach handling link asymmetry for wireless mesh access networks,' IEEE Transactions on Vehicular Technology, vol. 60, no. 3, pp. 1045-1058, Mar. 2011. https://doi.org/10.1109/TVT.2011.2106172.

[10] Peng Zhao, Xinyu Yang, Wei Yu, and Xinwen Fu, "A Loose-VirtualClustering based Routing for Power Heterogeneous MANETs," IEEE transactions on vehicular technology, vol. 62, no. 5, June 2013 https://doi.org/10.1109/TVT.2012.2237556.

[11] Abolhasan, M., J. Lipman, and J. Chicharo, "A routing strategy for heterogeneous mobile ad hoc networks", In Emerging Technologies: Frontiers of Mobile and Wireless Communication, 2004. Proceedings of the IEEE 6th Circuits and Systems Symposium on 2004

[12] W. Erik et al., "Improving routing performance in wireless ad hoc networks using cross-layer interactions", Ad Hoc Networks, Vol. 5, No. 5, pp. 579-599, 2007 . https://doi.org/10.1016/j.adhoc.2006.03.003.

[13] K. Xu, X. Hong, M. Gerla, "An Ad Hoc Network with Mobile Backbones," Proc. of IEEE ICC 2002, New York, NY, Apr. 2002.

[14] V. Ramasubramanian, et al., "Providing a Bidirectional Abstraction for Unidirectional Ad Hoc Networks," Proc. of IEEE INFOCOM 2002, 2002

[15] P. Sinha and S. Krishnamurthy, "Scalable Unidirectional Routing with Zone Routing Protocol (ZRP) Extenstions for Mobile Ad hoc Networks," Proc. of WCNC, Chicago, Sep. 2000.

[16] L. Bao and JJ Garcia-Luna-Aceves, "Link State Routing in Networks with Unidirectional Links," Proc. of IEEE ICCCN 1999, Boston, MA

[17] A. Avudainayagam, Y. Fang, and W. Lou, "DEAR: A device and energy aware routing protocol for heterogeneous ad hoc networks," J. Parallel Distributed Comput., vol. 63, no. 2, pp. 228-236, Feb. 2003. https://doi.org/10.1016/S0743-7315(02)00066-7.

[18] Mehran Abol hasan, Tadeusz Wysocki, Eryk Dutkiewicz, "A review of routing protocols for mobile ad hoc networks", Elsevier, Vol. 29, No. 1, 2004, pp. 23-33

[19] Djamel F. Hadj Sadok et al. , "On the performance of heterogeneous MANETs", Wireless Networks, Springer, Vol. 21, No. 1, pp 139-160, January 2015. https://doi.org/10.1007/s11276-014-0777-Z

[20] Wang, J.N., Narula-Tam, A., Byan, R., "Interconnecting Heterogeneous MANET Networks at the Tactical Edge", Military Communications Conference (MILCOM), IEEE, pp. 1152-1159, Oct 2014
[21] M V Narayana, G Narsimha, SSVN Sarma, "Secure- ZHLS: Secure Zone Based Hierarchical Link State Routing Protocol using Digita Signature", International Journal of Applied Engineering Research ISSN 0973-4562 Volume 10, Number 9 (2015) pp. 22927-22940.

[22] M V Narayana, G Narsimha, SSVN Sarma, "Genetic - ZHLS Routing Protocol for Fault Tolerence and Load Balancing" Journal of Theoretical and Applied Information Technology, ISSN: 1992-8645 and E-ISSN: 1817-3195, 10 January 2016. Vol.83. No.1, pp. $72-80$

[23] M V Narayana, G Narsimha, SSVN Sarma, "SHA-ZHLS: Security Enhancement in MANETs using SHA Algorithm" Internationa Journal of Advanced Research in Computer and Communication Engineering, ISSN (Online) 2278-1021 \& ISSN (Print) 2319 5940, Vol. 5, Issue 2, February 2016, pp.59-65. 